\title{
Poster: Performance Evaluation of an Open-Source Audio-Video Bridging/Time-Sensitive Networking Testbed for Automotive Ethernet
}

\author{
Teng Andrea Xu $\mathrm{Xu}^{\ddagger}$, Florian Adamsky*, Ion Turcanu*, Ridha Soua*, Christian Köbel ${ }^{\dagger}$, \\ Thomas Engel*, and Andrea Baiocchi ${ }^{\ddagger}$, \\ * \{name.surname\}@uni.lu, SnT, University of Luxembourg, Luxembourg \\ † Christian_Koebelede.hrdeu.com, Honda R\&D Europe, Germany \\ $\ddagger$ xu.1607870@studenti.uniromal.it, andrea.baiocchi@uniromal.it, University of Rome Sapienza, Italy
}

\begin{abstract}
Automotive Ethernet (AE) is becoming more and more relevant to the automotive industry due to its support of emerging in-car applications, which have high bandwidth demands and stringent requirements in terms of latency and time synchronization. One of the standards under consideration for AE is IEEE 802.1 Audio Video Bridging (AVB)/Time Sensitive Networking (TSN) that provides deterministic data link layer and bounded latency to real-time traffic classes. So far, this protocol stack has only been evaluated using either simulations or proprietary and expensive platforms. In this paper, we design a real testbed system for $\mathrm{AE}$ using general-purpose singleboard computers and conduct experiments to assess the real-time performance of an open-source AVB/TSN implementation. Our preliminary results show that even under heavy load, AVB/TSN can fulfil the latency requirements of $\mathrm{AE}$ while keeping a constant latency variation.
\end{abstract}

\section{INTRODUCTION}

Nowadays, cars are becoming increasingly dependant on a broad range of embedded computers, sensors, cameras, and other advanced systems, to enable safe and comfortable journey for drivers and passengers. In addition, it is expected that self-driving cars will hit our roads in the coming years, which will require an increased number of advanced sensors and high resolution driver assistance camera systems. To integrate these new features and ensure the delivery of bandwidthhungry and delay-sensitive traffic, it is crucial to have reliable, deterministic and bandwidth-guarantee communication protocols. Current intra-car networks adopted by car manufacturers include Controller Area Network (CAN), Local Interconnect Network (LIN), Media Oriented Serial Transport (MOST), and FlexRay. The main limitation of these technologies is that, at the time of their conception, they were not tailored with the sharp rise of high-bandwidth and delay-sensitive applications. To support the above mentioned requirements, in-car networks have to undergo significant changes.

Automotive Ethernet (AE) is considered to be a potential candidate technology able to meet the new stringent automotive requirements, at the same time offering much higher capacity than current technologies. Several AE-based protocols are currently being considered, such as IEEE Audio Video Bridging (AVB)/Time Sensitive Networking (TSN) and TimeTriggered Ethernet (TTEthernet), which are compared in [1]. Among these, AVB/TSN is gaining more attention from both the research community and automotive industry. However, to the best of our knowledge, the implementation and evaluation of AVB/TSN has been carried out using either computer simulations [1] or by running experiments on expensive testbeds with proprietary hardware and software [2], [3].

The contribution of this paper is two-fold: (i) we design a real AVB/TSN testbed system based on OpenAvnu [4], the open-source reference implementation provided by the Avnu Alliance; (ii) we investigate the performances of AVB/TSN in terms of latency and delay variation (jitter), defined as in [5], under different traffic loads using this testbed. We demonstrate that the timing constraints of critical data traffic can be ensured even in presence of high levels of best-effort background traffic.

\section{BACKGROUND AND RELATED WORK}

AVB/TSN is an evolution of Ethernet proposed to provide a deterministic data link layer. It includes a set of technical standards developed initially by the IEEE 802.1 AVB Task Group since 2005, which was renamed to TSN Task Group in 2012 [6]. The main standards included in AVB/TSN are: 1) IEEE $802.1 \mathrm{AS}$ for timing and synchronisation; 2) IEEE 802.1Qat for online stream reservation; 3) IEEE 802.1Qav for forwarding and queuing enhancement; 4) IEEE 802.1BA for audio and video bridging systems. AVB/TSN supports two traffic classes with different end-to-end latency guarantees: $\leq$ $2 \mathrm{~ms}$ for class A traffic, and $\leq 50 \mathrm{~ms}$ for class B traffic.

Since AVB/TSN is hardware constrained, many studies have focused on simulations. For example, a comparison between TTEthernet and AVB was done in [1]. Lim et al. tested AVB performance under different additional network loads (up to $70 \%$ ) and multiple hops, by relying on OMNeT++based simulations [7]. Outside the simulation environment, a performance evaluation of AVB and IEEE 802.1Q using embedded systems in a single-switch network was done in [2], even though the authors do not specify the software used. This work has been extended in [3], where a multi-switch network has been considered.

Differently from existing studies, we build an experimental testbed for AE based on general-purpose single-board computers using an open-source implementation of AVB/TSN, namely OpenAvnu [4]. We provide insights into how to build such a system and run experiments to validate the main features of AVB/TSN. 


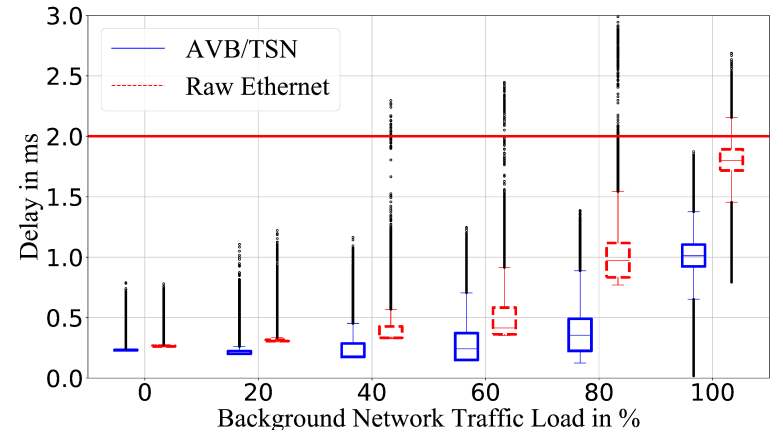

Fig. 1. Comparison of AVB/TSN (left blue solid boxplot) and raw Ethernet (right red dotted boxplot) in terms of latency. Payload size is $430 \mathrm{~B}$.

\section{Methodology and Evaluation}

Our testbed consists of two APU2C4 single-board computers equipped with Intel $囚$ I $2101 \mathrm{Gbit} / \mathrm{s}$ Network Interface Cards (NICs) supporting hardware timestamping, which is necessary for Generalized Precision Time Protocol (gPTP). Both devices run Ubuntu 16.04 GNU/Linux OS and the latest version (git commit $6788 \mathrm{f} 2 \mathrm{f}$ ) of OpenAvnu [4]. We reduced the NIC speed to $100 \mathrm{Mbit} / \mathrm{s}$, to bring the network more easily to its limits. To compare AVB/TSN with "normal" Ethernet, we developed a Python script ${ }^{1}$ to exchange raw Ethernet frames between the two computers. For the background traffic, we used iperf 3.5 to send best-effort UDP packets in parallel. The gPTP protocol, which is part of OpenAvnu, synchronizes the NIC clocks with a mean drift of $-13.4 \mathrm{~ns}$.

We carried out two experiments consisting of a Talker directly connected to the Listener, sending a $113.8 \mathrm{MB}$ video file. The goal of the first experiment is to verify if AVB/TSN is able to fulfil the maximum end-to-end latency constraint of $2 \mathrm{~ms}$ under different network traffic loads. To this end, we vary the background traffic between $0-100 \%(100 \% \widehat{=} 77.7 \mathrm{Mbit} / \mathrm{s})$ with a $22 \%$ step size and we measure the obtained latency. Results are depicted in Figure 1. Each boxplot represents the first and third quartiles as a box, the median value as a central line inside the box, and the minimum and maximum values with whiskers. The horizontal red line indicates the latency threshold for class A traffic. We can notice that both AVB/TSN and Ethernet have small delays for lower network traffic loads, while they present slightly increased delays for higher loads. However, it can be seen that AVB/TSN is able to always guarantee an end-to-end delay $\leq 2 \mathrm{~ms}$, independently from the considered network traffic load. Ethernet, on the other hand, fails to do so, especially under high traffic load in background. In particular, the percentage of measured delay values above the $2 \mathrm{~ms}$ threshold is $0.0069,0.02,0.062$ and $8.1 \%$ in case of $40,60,80$ and $100 \%$ levels of background best-effort traffic.

In the second experiment, we investigate the delay variance (jitter) for both AVB/TSN and Ethernet. Experimental results (average with $95 \%$ confidence interval) are shown in Figure 2. The mean delay variation ranges between $0.01-0.13 \mathrm{~ms}$ for AVB/TSN, while for Ethernet the range is in between 0.008-

\footnotetext{
${ }^{1}$ https://github.com/TengXu94/python_traffic_generator/
}

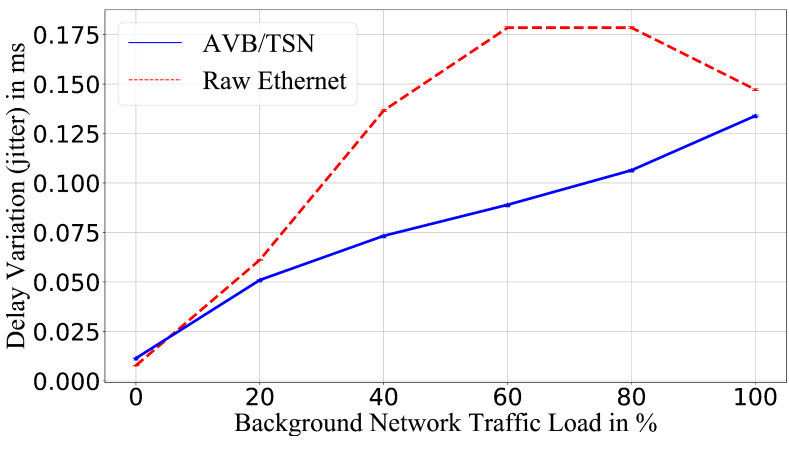

Fig. 2. Mean delay variation of AVB/TSN (blue solid line) and Ethernet (red dotted line) for different network traffic loads. Payload size is $430 \mathrm{~B}$.

$0.18 \mathrm{~ms}$. We can see that AVB/TSN is more resilient than Ethernet against different levels of traffic load, with a maximum difference of mean jitter of $0.12 \mathrm{~ms}$ for AVB/TSN, and $0.17 \mathrm{~ms}$ for Ethernet. The variance of the jitter in this experiment is 0.001 for AVB/TSN and 0.004 for raw Ethernet.

\section{CONCLUSion AND Future Work}

In this paper we designed a real and simple testbed for AE based on OpenAvnu, an open-source implementation of AVB/TSN. We used this testbed to investigate and compare the performances of AVB/TSN and Ethernet in terms of delay and jitter, under different network traffic loads. Our preliminary results show that, unlike Ethernet, AVB/TSN is able to fulfil the class A time constraints. Additionally, we found out that AVB/TSN has a lower jitter compared to Ethernet, which shows a higher variation. As a future work, we plan to extend the testbed and include switches in order to test different network topologies. Also, we plan to evaluate the AVB/TSN performance under multiple data streams with different priority levels.

\section{ACKNOWLEDGEMENT}

Funded by the Honda Initiation Grant Europe 2018.

\section{REFERENCES}

[1] T. Steinbach, H.-T. Lim, F. Korf, T. C. Schmidt, D. Herrscher, and A. Wolisz, "Tomorrow's In-Car Interconnect? A Competitive Evaluation of IEEE 802.1 AVB and Time-Triggered Ethernet (AS6802)," in Proceedings of the 76th IEEE Vehicular Technology Conference (VTC Fall). IEEE, Sep 2012, pp. 1-5.

[2] J. C. Moon, B. M. Cheon, J. D. Park, and J. W. Jeon, "Comparison of IEEE802. 1Q and IEEE802. 1AVB in in-vehicle network implemented in embedded system," in IEEE International Conference on Information and Automation. IEEE, Aug 2015, pp. 1491-1496.

[3] J. H. Seo and J. W. Jeon, "Comparison of IEEE802.1Q and IEEE802.1AVB in multi switch environment in embedded system," in 17th International Conference on Control, Automation and Systems (ICCAS), Oct 2017, pp. 489-493.

[4] A. Alliance, "OpenAvnu." [Online]. Available: https://github.com/AVnu/ OpenAvnu

[5] C. Demichelis and P. Chimento, "IP Packet Delay Variation Metric for IP Performance Metrics (IPPM)," RFC Editor, RFC 3393, Nov 2002. [Online]. Available: https://tools.ietf.org/html/rfc3393

[6] IEEE. Time-Sensitive Networking Task Group. [Online]. Available: http://www.ieee802.org/1/pages/tsn.html

[7] H.-T. Lim, D. Herrscher, M. J. Waltl, and F. Chaari, "Performance Analysis of the IEEE 802.1 Ethernet Audio/Video Bridging Standard," in Proceedings of the 5th International ICST Conference on Simulation Tools and Techniques, ser. SIMUTOOLS '12, 2012, pp. 27-36. 